\title{
DETECTION AND PHOTOMETRY OF RED GIANTS IN THE MAGELLANIC CLOUDS
}

\author{
Marc Aaronson \\ Steward Observatory, University of Arizona
}

\begin{abstract}
This review will focus on photometric observations of evolved stars in the Magellanic Clouds. Emphasis is placed on red and nearinfrared measurements, as these allow reasonable estimates to be made of bolometric magnitude and temperature for reliable placement in the physical HR diagram. The review is divided into three parts: field stars, cluster stars, and red variables; a summary of the surveys for objects in each of these areas is also given. Particular attention is drawn to the intermediate-age stellar population, as this component appears to be the primary tracer of the star formation rate and chemical enrichment history of the Clouds.
\end{abstract}

\section{INTRODUCTION}

Studies of the red stellar content in the Magellanic Clouds over the last ten years have led to new insight concerning both the star formation history in the Clouds and the evolutionary nature of the red giants themselves. A special incentive on both of these fronts has been provided by the discovery and subsequent photometry of large numbers of carbon stars in both the field and clusters of the Clouds. These stars appear to be signposts for a metal-poor, intermediate-age population which may in fact be the dominant stellar component in both the Large and Small Cloud.

The review that follows is divided into three sections on field stars, cluster stars, and red variables. Each part begins with a brief summary of the various survey results, followed by a discussion of the key photometric observations. For the latter, measurements at nearinfrared wavelengths are emphasized. Major advances in instrumentation have occurred since the last symposium on the Clouds, and photometry to the few percent level at JHK wavelengths is now routine on large telescopes for evolved Cloud stars near or brighter than the first giant branch tip. Such photometry permits accurate and straightforward estimation of bolometric magnitude, a quantity providing the critial 
link with stellar evolution theory. In this regard, working in the Clouds offers the well-known advantage of eliminating the (relative) distance uncertainties among objects so that, for instance, reliable luminosity functions can be determined. The IR also provides a better temperature estimator than can be obtained from optical photometry alone, although as mentioned further below the precise correspondence with effective temperature still remains a somewhat thorny issue.

Finally, it should be noted that the discussion here is confined largely to observational aspects of the red giants. The reader is referred to the reviews elsewhere in this volume by Mould (1983) for an extended treatment of theoretical comparisons, and by Humphreys (1984) for a discussion of the red supergiants.

\section{FIELD RED GIANTS}

In order to study the red giants we must first find them. With regard to the field, while two-color photometry has been employed to isolate red stars (e.g. Walker et al. 1969, Tifft and Snell 1971, Mendoza and Gomez 1973), it has been of limited use for detailed study of the stars themselves. Of more value have been objective prism surveys carried out with Schmidt-type telescopes and "grism" surveys with 4-m class telescopes, the latter reaching considerably fainter than the former, but at the cost of wide area coverage.

The most important objective prism surveys for the LMC have been those of Sanduleak and Philip (1977), who report $\sim 600$ M supergiants and $\sim 470$ carbon stars; Westerlund et al. (1978), who report $\sim 300 \mathrm{C}$ stars (most of which do not overlap with the Sanduleak and Philip sample, possibly owing to the use of red $\mathrm{CN}$ bands rather than $\mathrm{C}_{2}$ bands as the discriminating feature); and Westerlund et al. (1981), who report $\sim 500 \mathrm{M}$ supergiants and $\sim 600 \mathrm{M}$ giants. These surveys cover nearly the entire LMC area, but apparently sample only the more luminous carbon and late-M giants. An additional list of $\sim 1000$ red stars in selected LMC regions obtained from ultra-low dispersion spectra is given by Bappu et al. (1978 and references therein). For the SMC, objective prism plates have been primarily used to identify red supergiants, of which 500 are listed by Sanduleak (1975 and references therein) and Azzopardi and Vineau (1975). More recent objective prism surveys of Rebeirot et al. (1983) for the LMC and Prévot et al. (1983) for the SMC have led to detection of $\sim 200$ additional red supergiants in each Cloud.

Field grism surveys have been conducted by Blanco et al. (1978 and references therein), Blanco et al. (1980), and Richer and Westerlund (1983). A new Cloud grism survey is also underway by Westerlund (1983) and collaborators. Although some warmer-temperature, weak-banded carbon stars may be missed, these efforts nevertheless appear to reach well below the peak in the carbon star and late-M giant luminosity functions, providing good samples for statistical investigation. The most ambitious effort to date and the one whose results we shall briefly 
summarize is that of Blanco and McCarthy (1983). These authors have obtained grism plates of 37 SMC and 52 LMC selected areas, reaching from the center to the periphery of these systems. In the SMC, they identify some $\sim 860$ C stars, $\sim 60$ stars of type M6 or later, 130 M5 stars, and $\sim 1060$ M2-M4 stars; while in the LMC, the corresponding numbers are $\sim 1040, \sim 480, \sim 820$, and $\sim 4440$.

Blanco and McCarthy (1983) use their remarkable sample to examine the surface brightness distribution of the carbon stars, which they find correlates reasonably well with the red isophotes of de Vaucouleurs (1957). They estimate the total number of $C$ stars to be $\sim 11,000$ and $\sim 2,900$ in the LMC and SMC, respectively. These numbers are quite interesting because their ratio is comparable to that of the Clouds' integrated luminosities, suggesting a basic similarity in star formation history. For example, the work of Butcher (1977) and Stryker and Butcher (1981) implies that a major and possibly dominant star formation event took place in the LMC $2-4$ Gyr ago. Perhaps the similarity in the carbon star number (and luminosity function as discussed below) between the LMC and SMC is reflective of a similar event having occurred in the Small Cloud.

Blanco and McCarthy (1983) also find the ratio of $C$ to $M 6$ and latter types to be $\sim 2$ for the LMC and $\sim 14$ for the SMC. These ratios become $\sim 0.2$ and $\sim 0.6$ when stars as early as type M2 are considered. The difference in $C / M$ ratio is in accord with what is known about the relative metallicities of the Clouds. In particular, a decrease in abundance is expected to (a) diminish the number of late $M$ stars via decreasing TiO absorption and warming of the giant branch; and (b) increase the number of $C$ stars through the need to dredge up less carbon (e.g. Iben 1982) and the possible increase in efficiency of the dredge-up process itself (Wood 1981). Given that the relative numbers of Large and Small Cloud $C$ stars are the same, the former mechanism would seem to be more important in explaining the change in $\mathrm{C} / \mathrm{M}$ ratio, although this conclusion rests on the plausible yet unproven assumption that the mean carbon star ages in the Small and Large Cloud are similar. A final result of Blanco and McCarthy worth mentioning is the radial dependence of the $\mathrm{C} / \mathrm{M} 6+$ ratio, which is constant over the face of the LMC, but apparently changes from $\sim 19$ to $\sim 5$ in going from the center of the edge of the SMC. While small number statistics may account for part of the latter effect, it nevertheless appears to be real. The explanation is unclear, but a naive interpretation would be that metallicity of the intermediate-age population increases from the center outwards, contrary to expectations.

The general completeness of the Blanco and McCarthy survey has been verified by Frogel and Richer (1983), who scanned an area of the LMC Bar West field at 2.2 and $3.5 \mu \mathrm{m}$ in an unsuccessful search for "missing" AGB stars. Elias (1982) has conducted a similar mapping over selected Cloud areas at 1.6 and $10 \mu \mathrm{m}$, and the results of this work are awaited with interest, as are those from the orbiting telescope IRAS. One final comment on the grism effort is that, except for a few not- 
able exceptions (Blanco et al. 1981; Richer and Frogel 1980), it has not lead to the discovery of many $S$ stars. This appears to reflect the difficulty of distinguishing early $S$ from $M$ stars at low dispersion, rather than a real absence of the former, as shown by the recent spectroscopic work of Lloyd-Evans (1983a).

Extensive VRI photometry for field carbon stars in the Clouds has been published by Richer et al. (1979) and Richer (1981; see also Crabtree et al. 1976). The major JHK study has been that of Cohen et al. (1981), who use their data to convert the Blanco et al. (1980) I luminosity functions to true bolometric ones. The results are virtually identical in the LMC and SMC: both luminosity functions show a roughly Gaussian shape with a peak at $M_{\text {bol }} \sim-5 \mathrm{mag}$, and a range from -4 to $\sim-6$ mag. The two-fold discrepancy of these results with theoretical expectations (e.g. the absence of high-luminosity $C$ stars with $M_{b o l}<-6$, and the presence of so many low luminosity ones with $M_{b o l}>$ -5) has been discussed extensively in the literature.

The similarity in the LMC and SMC carbon star luminosity functions is perhaps surprising in view of the Clouds' known present day abundance differences. All else being equal, dredge-up theory would predict a lower mean luminosity for SMC carbon stars relative to LMC carbon stars (e.g. Iben 1982). The absence of this result suggests three possibilities: (a) the ease of conversion to a C star may bottom out at low metallicities when only one thermal pulse is required for the conversion to occur; (b) errors in relative distance moduli and/or differences in star formation history have conspired to mask the dredge-up effect and produce identical luminosity functions; or (c) the progenitor stars had similar metallicities. Evidence against the last suggestion comes from Cohen et al.'s (1981) study of the JHK twocolor diagram. These authors show that the loci of carbon stars are displaced such that $\mathrm{J}-\mathrm{H}$ is bluer at fixed $\mathrm{H}-\mathrm{K}$ as one progresses from the Milky Way to the LMC to the SMC. Cohen et al. cite blanketing changes in accord with present day metal differences as explanation for the effect. If so, it would seem that estimation of carbon star effective temperatures from $\mathrm{J}-\mathrm{K}$ colors is severely hampered for the Clouds, although Aaronson and Mould (1982) have shown that such color temperatures for galactic $C$ stars do provide a reliable match with the Ridgway et al. (1980) occultation results.

The field $M$ giants have not yet received quite the attention of the carbon stars. Recently, however, Frogel and Blanco (1983a) obtained IR photometry for a complete sample of known $M$ giants in the LMC Bar West field. These authors find evidence for a bi-modal distribution in the $(\mathrm{K}, \mathrm{J}-\mathrm{K})$ color-magnitude diagram which they suggest indicates the presence of two separate star formation bursts: the conventional $3-5$ Gyr old event and a more recent $10^{8}$ year old one. Additional supporting statistics for this conclusion via study of other Cloud fields would be welcome. 


\section{CLUSTER RED GIANTS}

The populous clusters provide the key for understanding of the chemical enrichment history in Clouds. The clusters also are the ultimate testing ground for theories of advanced stellar evolution, as both luminosities and ages of the red giants can (in principal) be directly determined. In this regard two questions are particularly relevant today. The first concerns the luminosity of the transition from $M$ to $C$ type and its dependence on age and metallicity. The second concerns the dependence of the maximum extent of the asymptotic giant branch (AGB) on these same quantities. This latter question is especially important for assessing the mechanism responsible for the so-called "missing" luminous AGB field stars (cf. Reid and Mould 1983), the primary choices here being variation in the star formation rate or dependence of stellar mass loss rate on age and abundance.

That the Cloud clusters contain very red stars has been known since the work of Arp and others in the late fifties (e.g. Arp 1958). However, it was sometime before the first of these were identified as carbon stars by Feast and Lloyd-Evans (1973); and only within the last few years have general surveys of cluster red stellar content been undertaken. Two-color photographic surveys at $V$ and $I$ have been published now by Lloyd-Evans (1980), Aaronson and Mould (1982), and Mould and Aaronson (1982). These efforts have largely concentrated on the red Cloud clusters, i.e., these having integrated $B-V$ color $\gtrsim 0.3$ mag. A new grism survey by Frogel and Blanco (1983b) is extending these results to bluer Cloud clusters.

The grism and two-color techniques are somewhat complementary for cluster investigations. In rich clusters the grism method is limited by crowding, an example being Blanco and Richer's (i979) work on NGC 419, which identified only 6 of the 15 or so carbon stars in this cluster found by Aaronson and Mould (1982). On the other hand, while the two-color method enables a complete exploration of the upper AGB, additional photometry or spectroscopy is required for stellar classification. Such observations have now been obtained for red stars in about a dozen SMC clusters and 35 LMC clusters, where $~ 70$ carbon stars, $20 \mathrm{M}$ stars, and $\sim 20 \mathrm{~S}$ stars have at present been located (Feast and Lloyd-Evans 1973; Mould and Aaronson 1979, 1980, 1982; Aaronson and Mould 1982, Lloyd-Evans 1980, 1983a,b; Bessell et al. 1981, 1983; Blanco and Richer 1979; Danks 1982). IR photometry has been secured for most of these stars and can be found in the cited references and additionally in Frogel et al. (1980) and Frogel and Cohen (1982).

A major photometric result to come out of the above efforts has been the demonstration that most of the red Cloud globulars have extended giant branches reaching well above the first giant branch tips of galactic globulars. This can be taken as prima facie evidence that the majority of the red clusters are of intermediate age. A similar conclusion has been reached by Rabin (1982) from spectrophotometric 
data, and by Searle et al. (1980) from integrated cluster photometry on the Gunn four-color system. A question of current interest is just how many of the Cloud clusters are really as old as galactic globulars? In particular, five out of six Searle et al. (1980) SWB type VII clusters appear to have extended giant branches, including such famous "old" objects as NGC 121, 1841, and 2257 (Mould and Aaronson 1982; Frogel and Cohen 1982). Deep color-magnitude diagrams and accurate metallicities will ultimately settle this issue, but in the meantime, it would be nice to have spectroscopic confirmation of membership for the one or two key stars in the older clusters.

Carbon stars populate the upper AGB and define the tip luminosity in most of the intermediate-age globulars. Their luminosity function appears similar to that of the field $C$ stars, with a range in $M_{b o l}$ from $\sim-4.2$ to $\sim-5.6$. Except for the peculiar variable V8 in NGC 121, C stars have not been located in SWB type VII or types I-III clusters, being apparently confined between types III-IV to types VI. This conclusion for the early SWB types rests at present primarily on the integrated infrared colors (see below) of Persson et al. (1983), and detailed exploration of the red stellar content in these younger clusters is awaited.

The JHK two-color diagram has proven to be an effective way of selecting carbon from non-carbon stars (cf. Figures 2 and 3 in Aaronson and Mould 1982), owing to the generally much redder colors of the former. There is, however, a gray area in which the photometric colors and luminosities overlap. It is precisely this region where Bessell et al. (1983) and Lloyd-Evans $(1983 a, b)$ have located a number of $S$ stars, dramatically confirming the association of these objects with the transition region between $M$ and $C$ types. Lloyd-Evans $(1983 a, b)$ and Frogel and Blanco (1983b) have explored this transition zone in detail. Their principle conclusions can be summarized as follows: (a) Most of the non-carbon cluster stars above an $\mathrm{M}_{\mathrm{bol}}$ of -4.3 are $\mathrm{S}$ stars. (b) The ratio of $\mathrm{S}$ to $\mathrm{C}$ stars is greater in the younger groups. (c) The $\mathrm{C}$ stars are generally not found less luminous than 0.2 mag from the brightest non-carbon stars. (d) The transition from $M \rightarrow S$ and $S \rightarrow C$ appears brighter in the younger groups, ranging from $\mathrm{M}_{\mathrm{bol}} \sim-4.5 \mathrm{mag}$ at SWB type VI to $M_{\text {bol }} \sim-5 \mathrm{mag}$ at SWB type IV. These results are in qualitative agreement with dredge-up theory; in particular, younger and more massive stars are expected to have to undergo more thermal pulses and thus climb higher on the AGB before turning themselves into carbon stars. Whether the results are in detailed quantitative agreement with theory must await accurate ages and metallicities for the clusters and fuller explorations of the transition region.

The tip luminosity has been used by Mould and Aaronson (1982 and references therein) as a cluster age estimator. For sparse clusters only an upper limit can be obtained, but for rich clusters with wellpopulated AGB's a specific age is deducible. Figure 1 illustrates the correspondence between SWB type and observed AGB extent for the sample from Mould and Aaronson. A rough correlation is present, but there is 
considerable spread at a given SWB type, with an apparent turnover between types IV and VI. The appearance of Figure 1 can be understod in part because (a) Mbol, max provides only an upper age limit; (b) several lower luminosity clusters populate the lower border of the type $V$ and VI lines, and stochastic effects may result in the complete absence of upper AGB stars in them; and (c) the SWB types themselves may have a wide spread in age at fixed type. Primarily because of the last reason, the results in Figure 1 cannot be used to meaningfully gauge $\mathrm{M}_{\mathrm{bol}}$, $\max$ as an age estimator, as some authors have attempted. The true test will come when more main sequence turn-off ages become known. The presently available turn-off ages appear to correspond well to $\mathrm{Mbol}_{\mathrm{bax}}$ for clusters older than $1 \mathrm{Gyr}$, but poorly for clusters younger than this (Mould 1984); the latter result if upheld may ultimately require modification of the adopted mass-loss rates. In any event, the addition of earlier SWB types to Figure 1 will clearly be of importance.

The cluster carbon stars in the SMC appear to have in the mean bluer JHK colors than these in the LMC, similar to the above-mentioned results found by Cohen et al. (1981) for the field $C$ stars. This may again reflect a lower mean abundance in the SMC clusters as compared with those in the LMC. Aaronson and Mould (1982) have also noted a peculiarity of the non-carbon cluster stars involving their tendency to deviate substantially from the $(J-K, V-K)$ two-color relation characterizing galactic field and cluster giants. The explanation for this effect remains unclear, but its presence obviously complicates the derivation of believable effective temperatures.

In closing this section, we mention the dramatic influence that the upper $A G B$ and particularly carbon stars have on the integrated infrared colors of the red Cloud clusters (Persson et al. 1983). This 
effect may be of use for locating intermediate-age clusters in other nearby galaxies, and may also be of importance for understanding the IR colors of high-z systems. However, it should be noted that Blanco and McCarthy (1983) have shown the total field bolometric contribution from carbon stars to be only $\sim 3 \%$ for the LMC and $\sim 4 \%$ for the SMC. Because of their extreme redness, the $C$ stars have much less effect on integrated cluster colors either in the optical (e.g. van den Berg 1981 and references therein) or UV (de Boer 1981).

\section{RED VARIABLES}

Luminous long period variables in the Clouds are rare, only 24 having been identified by Payne-Gaposchkin and Gaposchkin (1966) in the SMC, and 46 having been identified by Payne-Gaposchkin (1971) in the LMC, although these authors have located a number of red irregular and semi-regular variables as well. The frequency of lower luminosity red variables is less clear, as the only published results remain those of Lloyd-Evans $(1978 a, b)$ for selected regions near the SMC clusters NGC 371 and 419. The data base for investigation of these objects is thus sparse, and additional searches for them such as those of Lloyd-Evans (1984), Paltoglou et al. (1984), and Reid (1983) are most welcome. Earlier reviews of the variables are given by Lloyd-Evans (1975), Feast (1973), and references therein.

Infrared photometry of the red Cloud variables can be particularly valauable for studying their pulsational properties and determining pulsational masses, and possibly as well for application to the extragalactic distance scale, since such stars are measurable out to quite large moduli. Until recently, work in these areas has largely been carried out by the South African Group (Catchpole and Feast 1981, Feast et al. 1980, Glass 1979, Glass and Feast 1982, and Glass and Lloyd-Evans 1981). Two principal results that have come from these efforts are (a) the period-luminosity relation for supergiants has been shown to be consistent with the theoretical relation for massive stars undergoing core helium burning; and (b) the Mira variables are favored as being first overtone pulsators. Unfortunately, this latter conclusion rests with the still considerably uncertain effective temperature calibration. (Photometry of individual variables of interest has also been presented by Elias et al. 1980, Bessell and Wood 1983, and Frogel 1983.)

Recently, studies by Wood et al. (1981, 1983) appear to have somewhat clarified the situation with regard to the red Cloud variables. Figure 6 in the last reference shows a clear segregation of the variables into two groups; these are identified as luminous small- amplitude $(\Delta \mathrm{K} \sim 0.25 \mathrm{mag})$ supergiant core helium burners, and lower-luminosity large amplitude $(\Delta K \sim 0.5-1 \mathrm{mag})$ AGB stars. The latter extend up to the AGB limit at $M_{b o l} \sim-7 . \quad S$ stars are found $u p$ to this limit and indicate that dredge-up is occurring at these luminosities, but the absence of carbon variables above $\mathrm{M}_{\mathrm{bol}} \sim-6$ lead Wood et al. to conclude that dredged-up carbon is being eliminated through $\mathrm{CN}$ cycling. 
It is interesting that in their Figure 6 SMC variables are seen with the same P-L properties as the LMC Miras (which in turn appear similar to the 47 Tuc variables). Hence, although SMC Miras have not been specifically identified as such, stars which may correspond to them do appear to exist.

The results of Wood et al. (1983) do not on the face of it bode well for distance scale measurements to other galaxies. In particular, the supergiant and AGB tracks are well separated by $\sim 1 \mathrm{mag}$ at the long period end and $\sim 2 \mathrm{mag}$ at the short period end. P-L relations alone cannot disentangle the two tracks. $\mathrm{P}-\mathrm{L}-\mathrm{A}$ relations involving amplitude may help, though, and need to be explored. Even so, the spread in magnitude at fixed period on the AGB track is quite large at $\sim 1$ mag. It does not seem that two $\mathrm{P}-\mathrm{L}$ relations would be sufficient to characterize this spread, as has been suggested by Feast (1981), but further investigation of this question via $\mathrm{P}-\mathrm{L}-\mathrm{A}$ and $\mathrm{P}-\mathrm{L}-\mathrm{C}$ relations is clearly required.

Preparation of this article was partially funded by NSF grant AST 81-17365. The author would also like to thank the organizers of IAU Symposium No. 108 for travel support.

\section{REFERENCES}

Aaronson, M., and Mould, J.: 1982, Ap. J. Suppl., 48, 161. Arp, H. C.: 1958, A. J., 63, 273 .

Azzopardi, M., and Vineau, J.: 1975, Astr. Ap. Suppl., 22, 285.

Bappu, M. K. V., Parthasarathy, M., and Searia, K. K.: 1978, Kodaikanal Obs. Bull., Ser. A., 2, 184 .

Bessell, M. S., and Wood, P.R.: 1983, M.N.R.A.S., 202, 31p.

Bessell, M. S., Wood, P. R., and Lloyd-Evans, T.: 1981, Proc. A.S.A., $4,201$.

$$
\text { : 1983, M.N.R.A.S., 202, 59. }
$$

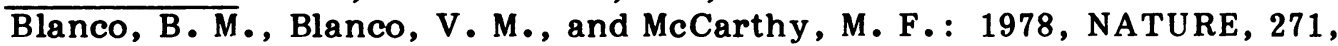
638.

Blanco, V. M., Frogel, J. A., and McCarthy, M. F.: 1981, Pub. A.S.P., 93,532 .

Blanco, V. M., and McCarthy, M. F.: 1983, preprint.

Blanco, V. M., McCarthy, M. F., and Blanco, B. M.: 1980, Ap. J., 242 , 938.

Blanco, V. M., and Richer, H. B.: 1979, P.A.S.P., 91, 659.

Butcher, H.: 1977, Ap. J., 216, 372.

Catchpole, R. M., and Feast, M. W.: 1981, M.N.R.A.S., 197, 385 .

Cohen, J. G., Frogel, J. A., Persson, S. E., and Elias, J. H.: 1981, Ap. J., 249, 481 .

Crabtree, P. R., Richer, H. B., and Westerlund, B. E.: 1976, Ap.J. Letters), 203, L81.

Danks, A. C.: 1982, Astr. Ap., 106, 4 .

de Boer, K. S.: 1981, in IAU Coll. No. 68, "Astrophysical Parameters for Globular Clusters", eds. A. G. D. Philip and D. S. Hayes (Schenectady: L. Davis Press), p. 3 . 
de Vaucouleurs, G.: 1957, A. J., 62, 69.

Elias, J. H.: 1982, private communication.

Elias, J. H., Frogel, J. A., and Humphreys, R. M.: 1980, Ap.J. (Letters), 242 , L13.

Feast, M. W.: 1973, in IAU Symp. No. 59, "Stellar Instability and Evolution", eds. P. Ledoux, A. Noels, and A. W. Rodgers (Dordrecht: Reidel), p. 93.

: 1981, in "The Most Massive Stars", eds. S. D'odorico, D. Baade, and K. Kjar (Garching: ESO), p. 217.

Feast, M. W., Catchpole, R. M., Carter, B. S., and Roberts, G.: 1980, M.N.R.A.S., 193,377 .

Feast, M. W., and Lloyd-Evans, T.: 1973, M.N.R.A.S., 164, 15p.

Frogel, J. A.: 1983, Ap. J., 272, 116 .

Frogel, J. A., and Blanco, V. W.: 1983a, preprint.

: 1983b, presented at IAU Symp. No. 105, "Observational Tests of Stellar Evolution Theory".

Frogel, J. A., and Cohen, J. G.: 1982, Ap. J., 253, 580 .

Frogel, J. A., Persson, S. E., and Cohen, J. G.: 1980, Ap. J., 239, 495.

Frogel, J. A., and Richer, H. B.: 1983, Ap. J., in press.

Glass, I. S.: 1979, M.N.R.A.S., 186,317 .

Glass, I. S., and Feast, M. W.: 1982, M.N.R.A.S., 199, 245.

Glass, I. S., and Lloyd-Evans, T.: 1981, NATURE, 291, 303.

Humphreys, R. M.: 1984, in IAU Symp. No. 108, "Structure and Evolution of the Magellanic Clouds", this volume, p. 145.

Iben, I.: 1982, Ap. J., 260, 821 .

Lloyd-Evans, T.: 1975, in "Variable Stars and Stellar Evolution", eds.

V. Sherwood and L. D. Plaut (Doredrecht: Reidel), p. 531.

: $1978 \mathrm{a}, \mathrm{M} . \mathrm{N} \cdot \mathrm{R} \cdot \mathrm{A} . \mathrm{S} ., 183,305$.

: 1978 b, M.N.R.A.S., 183,319 .

$1980, M . N . R . A . S ., 193,87$.

1983a, M.N.R.A.S., in press.

1983b, M.N.R.A.S., submitted.

1984 , presented at IAU Symp. No. 108, "Structure and

Evolution of the Magellanic Clouds", this volume, p. 217.

Mendoza, E. E., and Gomez, T.: 1973, Pub. A.S.P., 85, 439.

Mould, J. R.: 1984 in IAU Symp. No. 108, "Structure and Evolution of

the Magellanic Clouds", this volume, p. 195.

Mould, J., and Aaronson, M.: 1979, Ap. J., 232, 421.

: 1980, Ap. J., 240, 464.

: 1982, Ap.J., 263, 629.

Paltoglou, G., Wood, P. R., and Bessell, M. S.: 1984, presented at IAU

Symp. No. 108, "Structure and Evolution of the Magellanic Clouds",

this volume, p. 219.

Payne-Gaposchkin, C. H.: 1971, Smith Contr. Ap., No. 13.

Payne-Gaposchkin, C.H., and Gaposchkin, S.: 1966, Smith. Contr. Ap., No. 9 .

Persson, S. E., Aaronson, M., Cohen, J. G., Frogel, J. A., and Matthews, K.: 1983, Ap. J., 266, 105.

Prévot, L., Martin, N., Maurice, E., Rebeirot, E., and Rousseau, J.: 1983, Astr. Ap. Suppl., 53, 255. 
Rabin, D.: 1982, Ap. J., 261, 85 .

Rebeirot, E., Martin, N., Miares, P., Prévot, L., Robin, A., Rousseau, J., and Peyrin, Y.: 1983, Astr. Ap. Suppl., 51, 277.

Reid, N.: 1983, privately communicated by I. S. Glass.

Reid, N., and Mould, J. R.: 1983, in preparation.

Richer, H. B.: 1981, Ap. J., 243, 744.

Richer, H. B., and Frogel, J. A.: 1980, Ap. J. (Letters), 242, L9.

Richer, H. B., Olander, N., and Westerlund, B. E.: 1979, Ap. J., 230, 724.

Richer, H. B., and Westerlund, B. E.: 1983, Ap. J., 264, 114.

Ridgway, S. T., Jacoby, G. H., Joyce, R. R., and Wells, D. C.: 1980, in IAU Coll. No. 59, "Physical Processes in Red Giants", eds. I. Iben and A. Renzini (Dordrecht: Reidel), p. 47.

Sanduleak, N.: 1975, Astr. Ap., 39, 461.

Sanduleak, N., and Philip, A. G. D.: 1977, Pub. Warner Swasey Obs., 2, No. 5.

Searle, L., Wilkinson, A., and Bagnuolo, N.: 1980, Ap. J., 239, 803. Stryker, L., and Butcher, H.: 1981, in IAU Coll. No. 68, "Astrophysical Parameters for Globular Clusters", eds. A. G. D. Philip and D. S. Hayes (Schnectady: L. Davis Press), p. 255.

Tifft, W. G., and Snell, C. M.: 1971, M.N.R.A.S., 151, 365.

van den Bergh, S.: 1981, Astr. Ap. Suppl., 46, 79.

Walker, M. F., Blanco, V. M., and Kunkel, W. E.: 1969, A. J., 74, 44.

Westerlund, B. E.: 1983, private communication.

Westerlund, B. E., Olander, N., and Hedin, B.: 1981, Astr. Ap. Suppl., 43,267 .

Westerlund, B. E., Olander, N., Richer, H. B., and Crabtree, D. R.: 1978, Astr. Ap. Suppl., 31, 61.

Wood, P. R.: 1981, in IAU Coll. No. 59, "Physical Processes in Red Giants", eds. I. Iben and A. Renzini (Dordrecht: Reidel), p. 135.

Wood, P. R., Bessell, M. S., and Fox, M. W.: 1981, Proc. A.S.A., 4, 203. : 1983, Ap. J., 272, 99. 


\section{DISCUSSION}

Hesser: Is NGC2257 one of the SWB VII clusters that you suspect has an extended giant branch? I ask because there is a poster paper by Hesser, McClure, and Harris (see this volume) reporting a new CMD based on CCD data reaching the upper $\simeq 1.5$ mags of the main sequence. The CMD is very similar to that of $\mathrm{M} 92$, as previously suggested by Stryker and as we also found from SIT vidicon photometry. NGC2257 also has a substantial RR Lyrae star population. Consequently I would be surprised if NGC2257 is younger than the Galactic globular clusters.

Aaronson: That's why membership of the key one or two stars needs to be checked. If these can be demonstrated to be members, then there is clearly something different going on in the giant branch evolution between the LMC and the Galaxy.

Frogel: In at least one SWB VII cluster the brightest star, which is apparently inore luminous than the brightest stars in Galactic globulars, is a close (about l") double. Both stars are of nearly equal magnitude anci color. Thus in at least this cluster ( I think it is NGC1841), the problem you mentioned with luminous stars goes away.

Aaronson: Good ! 